\title{
MODELLING AND TESTING OF THE PIEZOELECTRIC BEAM AS ENERGY HARVESTING SYSTEM
}

\author{
Andrzej KOSZEWNIK*, Krzysztof WERNIO* \\ *Bialystok University of Technology, Faculty of Mechanical Engineering, ul. Wiejska 45C,15-351 Bialystok, Poland \\ a.koszewnik@pb.edu.pl, werniokrzysztof@gmail.com
}

received 31 March 2016, revised 29 November 2016, accepted 2 December 2016

\begin{abstract}
The paper describes modelling and testing of the piezoelectric beam as energy harvesting system. The cantilever beam with two piezo-elements glued onto its surface is considered in the paper. As result of carried out modal analysis of the beam the natural frequencies and modes shapes are determined. The obtained results in the way mentioned above allow to estimate such location of the piezoactuator on the beam where the piezo generates maximal values of modal control forces. Experimental investigations carried out in the laboratory allow to verify results of natural frequencies obtained during simulation and also testing of the beam in order to obtain voltage from vibration with help of the piezo-harvester. The obtained values of voltage stored on the capacitor $C_{0}$ shown that the best results are achieved for the beam excited to vibration with third natural frequency, but the worst results for the beam oscillating with the first natural frequency.
\end{abstract}

Key words: Cantilever Beam, Piezo-Actuator, Energy Harvesting System, Modal Analysis

\section{INTRODUCTION}

Vibration-based energy harvesting has received a great attention in the past decade. Research motivation in this field is due to the reduced power requirement of small electronic components such as the wireless sensors used in structural health monitoring applications. Research in this area involves understanding the mechanics of vibrating structures, the behaviour of piezoelectric materials and the electric circuit theory. This promising way of powering small electronic components and remote sensors has attracted researches from different disciplines of mechanical and electrical engineering.

As described main editors of the book (Priya and Inman, 2009) exist three basic mechanisms of conversation vibration to electric energy: electromagnetic (Arnold, 2007; Williams and Yates, 1996), electrostatic (Roundy et al., 2002), and piezoelectric (Sodano et al., 2005). These transduction mechanisms in the last decade have been widely investigated by researches for vibrationbased energy harvesting. The literature of the last eight years shows that piezoelectric transduction has received the most attention for vibration to electricity conversation. Especially, it is shown in review articles (Anton and Sodano, 2007; Bai et al, 2015; Borowiec, 2015; Chen et al., 2006; Friswell et al., 2012; Tan et al., 2015) which the simulation and experimental results proved that vibration to energy conversation by piezoelectric might be used in many applications. In these references probem of non-linear of the piezoelectric elements is considered in order to achieve maximum harvested energy.

Typically, a piezoelectric harvester is a cantilever beam with piezo-ceramic layers located on the a vibrating shake. As a result of vibration of the beam the dynamic strain induced in the piezoceramic layers generates an alternating voltage output across the electrodes covering the piezo-ceramic layers. In many cases such approach allow to determine the mathematical model of the beam that can be used in many practical applications.
In the present paper problem of conversion vibration to electric energy for active cantilever beam is described. In considered case the beam is excited to vibration by the piezo-stripe actuator located on the top side of the beam in quasi-optimal location. As a result of applied sinusoidal excitation to the piezo-actuator with frequency equals natural frequency the voltage from the piezo-harvester is obtained. The experimental investigations carried out at the lab stand show how the excitation of the beam may influence on the voltage derived from the piezo-harvester located on the free end of the beam. In results of such analysis can see relationship between mechanical and electrical part of consider smart beam.

\section{MODELLING OF THE BEAM - ANALYTICAL APPROACH}

In this section the mathematical model of the cantilever beam with piezo-actuator and the piezo-harvester shown in Fig.1 is analytically formulated.

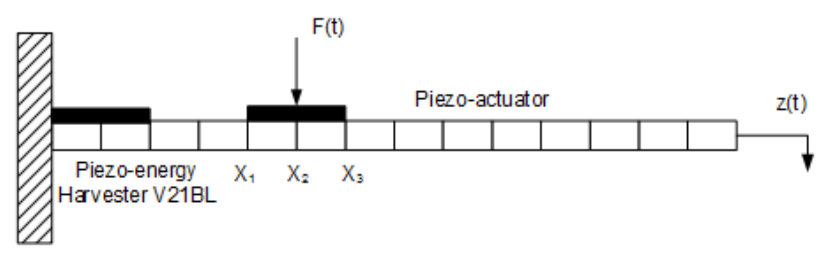

Fig. 1.The cantilever steel beam with the piezo-actuator and the piezo-harvester

The model of the beam for the first four lowest natural frequencies is determined for given actuator locations. In result of such assumed assumptions the equation of forced vibrations of the beam can be written as: 
$\frac{\partial^{4} y(x, t)}{d x^{4}}+a_{z}{ }^{2} \frac{\partial^{2} y(x, t)}{d t^{2}}=$
$\frac{1}{E_{b} I_{b}} F_{A}\left(-\delta\left(x-x_{1}\right)+2 \delta\left(x-x_{2}\right)-\delta\left(x-x_{3}\right)\right)$

where: $a_{z}=\sqrt{\frac{\rho_{b} A_{b}}{E_{b} I_{b}}}, \delta-$ Dirac function.

Eq.(1) has been rewritten after modal transformation to the following form:

$\frac{\partial^{4} y(x, t)}{d x^{4}}+a_{z}{ }^{2} \frac{\partial^{2} y(x, t)}{d t^{2}}=\sum_{n=1}^{\infty} F_{A n} U_{n}(x)$

where: $F_{A n}=F_{A}\left(-U_{n}\left(x_{1}\right)+2 U_{n}\left(x_{2}\right)-U_{n}\left(x_{3}\right)\right)-$ modal transversal force of the piezo-actuator.

Transformations presented in Eq.(2) lead to determine of the mode shapes of the beam. However before to do need consider the boundary conditions of the piezoelectric beam expressed in following form:

Fixed end:

$y(x, t)=0$ and $\left.\frac{\partial y(x, t)}{\partial x}\right|_{x=0}=0$

Free end: $\quad M(x, t)=\left.\frac{\partial^{2} y(x, t)}{\partial x^{2}}\right|_{x=l}=0$ and $F(x, t)=$ $\left.\frac{\partial^{3} y(x, t)}{\partial x^{3}}\right|_{x=l}=0$

As results of boundary conditions the displacement $y(x, t)$ is splitted into variable described in space $-U(x)$ and variable dependent in time $-T(t)$. Such approach leads to determining of the modes shapes $U_{n}(x)$ that it expressed in the following form (Kelly, 2007):

$$
\begin{aligned}
& U_{n}(x)=\left(\operatorname{sh} k_{n} l+\sin k_{n} l\right)\left(\operatorname{ch} k_{n} x-\cos k_{n} x\right)+ \\
& -\left(\operatorname{ch} k_{n} l+\cos k_{n} l\right)\left(\operatorname{sh} k_{n} x-\sin k_{n} x\right)
\end{aligned}
$$

where: $k_{n} l=\frac{2 n-1}{2} \pi$ for $n=1,2,3$.

The mechatronic system shown in Fig.1 is transformed to equivalent model that it is shown in Fig. 2 The obtained model is described by mass, spring, damper and the piezo structure energy storage system.

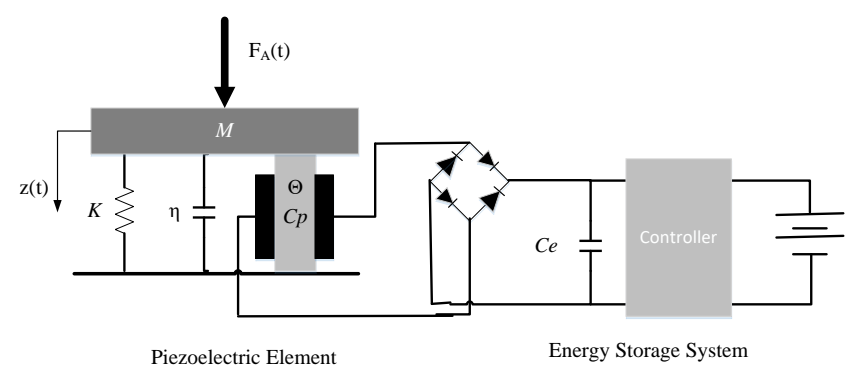

Fig. 2. The euqivalent model for a piezoelectric vibration energy harvesting system

As it can be seen in Fig. 2 the model consists a vibrating piezoelectric element which generates an $A C$ voltage which next is stabilize to DC voltage. Especially it is important in case of wireless sensors that they require such supply. The obtained in this way model is electromechanical system which it is expressed as:

$$
\left\{\begin{array}{l}
M \ddot{z}(t)+\eta \dot{z}(t)+K z(t)+\Gamma V_{p}(t) \\
I(t)=\Gamma \dot{z}(t)-C_{p} \dot{V}_{p}(t)
\end{array}\right.
$$

where: $M$ - effective mass of the system, $\eta$ - coefficient of damper, $K$ - effective stiffness of the system, $\Gamma$ - electromechanical coupling factor, $V_{p}(t)$ - the output voltage of equivalent system.

Moreover, above calculations lead to determine of the electromechanical behaviour of the beam. In this order need to define Generalized Electromechanical Coupling Factor known in the literature as GEMC factor $\Gamma$ that it shows relationship between mechanical and electrical parameters of the piezo-energy harvester element. This factor is expressed in the following form (Koszewnik et al., 2015):

$\Gamma^{2}=k_{31}{ }^{2} k_{p} C_{0}$

where: $k_{31}$ - coupling coefficient for length extensional, $k_{p}-$ stiffness of the piezoelectric material, $C_{0}$ - output capacitance.

Taking into account form of the GEMC factor described with Eq.(6) and the equation of maximum power output derived from harvester expressed as:

$P_{\text {OUT_MAX }}=\left.\frac{F_{A}^{2}}{8 d}\right|_{\begin{array}{l}\omega=\omega_{n} \\ d=d_{e}=d_{m}\end{array}}$

where: $P_{\text {OUT_MAX }}$ - the maximum value of the power output, $\omega_{n}$ - the natural frequency of the beam, $m d_{e}, d_{m} m$ - the electrical and mechanical damping.

Quasi-static electro-mechanical behavior of the beam can be described in a following form:

$\left[\begin{array}{l}F_{A} \\ Q\end{array}\right]=\left[\begin{array}{ll}k_{b} & \Gamma \\ \Gamma & -C_{0}\end{array}\right]\left[\begin{array}{l}\Delta x \\ U\end{array}\right]$

where: $F_{A}$ - the force applied to the structure, $Q$ - the charge on the piezo, $k_{b}$ - the stiffness of the beam, $\Delta x$ - elongation, $U$ - output voltage.

In case of assumed perfect link between the piezoelectric layer and the beam $(\Delta x=z)$ is possible transformation of Eq.(8) to form:

$\left[\begin{array}{l}F_{A} \\ Q\end{array}\right]=\left[\begin{array}{ll}k_{b} & \Gamma \\ \Gamma & -C_{0}\end{array}\right]\left[\begin{array}{l}Z \\ U\end{array}\right]$

where: $z$ - the displacement of free end of the beam.

Finally, the force $F_{A}$ applied to the beam structure can be expressed as sum of mechanical part of the beam caused by mass velocity and electrical part caused by mounted piezoelectric elements expressed in the following form:

$F_{A}=k_{b} z+\Gamma U$

\section{MODELLING OF THE BEAM - NUMERICAL APPROACH}

The cantilever beam with two rectangular piezoelectric stripes (an actuator and a harvester) glued onto its surface is shown in Fig.1. The steel beam has the dimension of $25 \times 400 \times 1.5 \mathrm{~mm}$, while the actuator QP20N and the piezo-harvester V21B are single piezoelectric stripes of $25 \times 50 \times 0.76 \mathrm{~mm}$ and $16.6 \times 36.6 \times 0.5$, respectively. The parameters of the cantilever beam are collected in Table.1. The model of the piezo-actuator is considered as a "static coupled model" (Gosiewski and Koszewnik, 2007) with 
the difference that the piezo-stripe is divided into two equal segments. As a result the bending moment generated by the piezoactuator is represented by a couple of opposite site forces concentrated at the segment's edge as it is shown in Fig.1 .

Tab. 1. Parameters of a cantilever beam

\begin{tabular}{|l|l|}
\hline Parameter & Value \\
\hline Length $/$ & $0.4 \mathrm{~m}$ \\
\hline Width $b$ & $0.025 \mathrm{~m}$ \\
\hline Thickness $t$ & $0.0015 \mathrm{~m}$ \\
\hline Youngs modules $E_{b}$ & $210 \mathrm{GPa}$ \\
\hline Density $\rho_{b}$ & $7800 \mathrm{~kg} / \mathrm{m}^{3}$ \\
\hline
\end{tabular}

\section{MODAL ANALYSIS OF THE BEAM}

Modal analysis of the piezoelectric beam is divided into two steps. In the first step of simulations the natural frequencies and mode shapes of the beam are determined. In this order FEM model of the beam shown in Fig. 1 is used and next investigated with help of Ansys software. In results of these analysis the first four lowest natural frequencies and mode shapes are achieved. The obtained results are shown in Fig. 3 and Tab. 2.

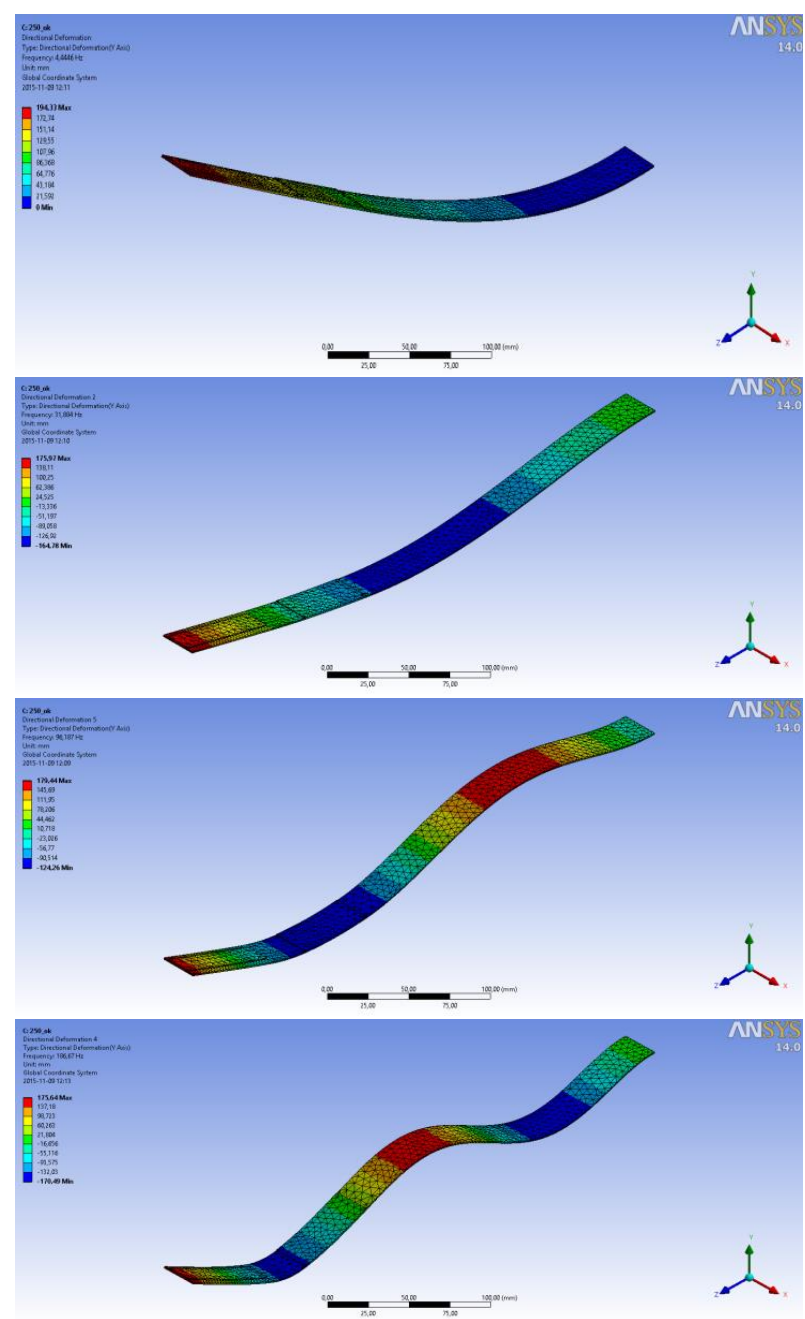

Fig. 3. The first four lowest mode shapes of the cantilever beam. The red colour denotes maximum displacement of the beam, but the blue line denotes minimum displacement
Tab. 2. First four natural frequencies of the piezoelectric beam

\begin{tabular}{|c|c|c|c|c|}
\hline & 1st $^{\text {st }}$ mode & $2^{\text {nd }}$ mode & 3 $^{\text {rd }}$ mode & 4 $^{\text {th }}$ mode \\
\hline $\begin{array}{c}\text { Natural } \\
\text { frequency } \\
\text { of the beam } \\
{[\mathrm{Hz}]}\end{array}$ & 7.13 & 48.34 & 141.9 & 288.2 \\
\hline
\end{tabular}

Next, the investigations is focused to determine such location of the piezo-actuator where this piezo generates maximal control forces. In this case the piezo is shifted onto surface on the beam from fixed end to free end of the beam with step equal 1/2 length of the piezo. For each location of the piezo-actuator the modal control forces are calculated and shown in Fig. 4.
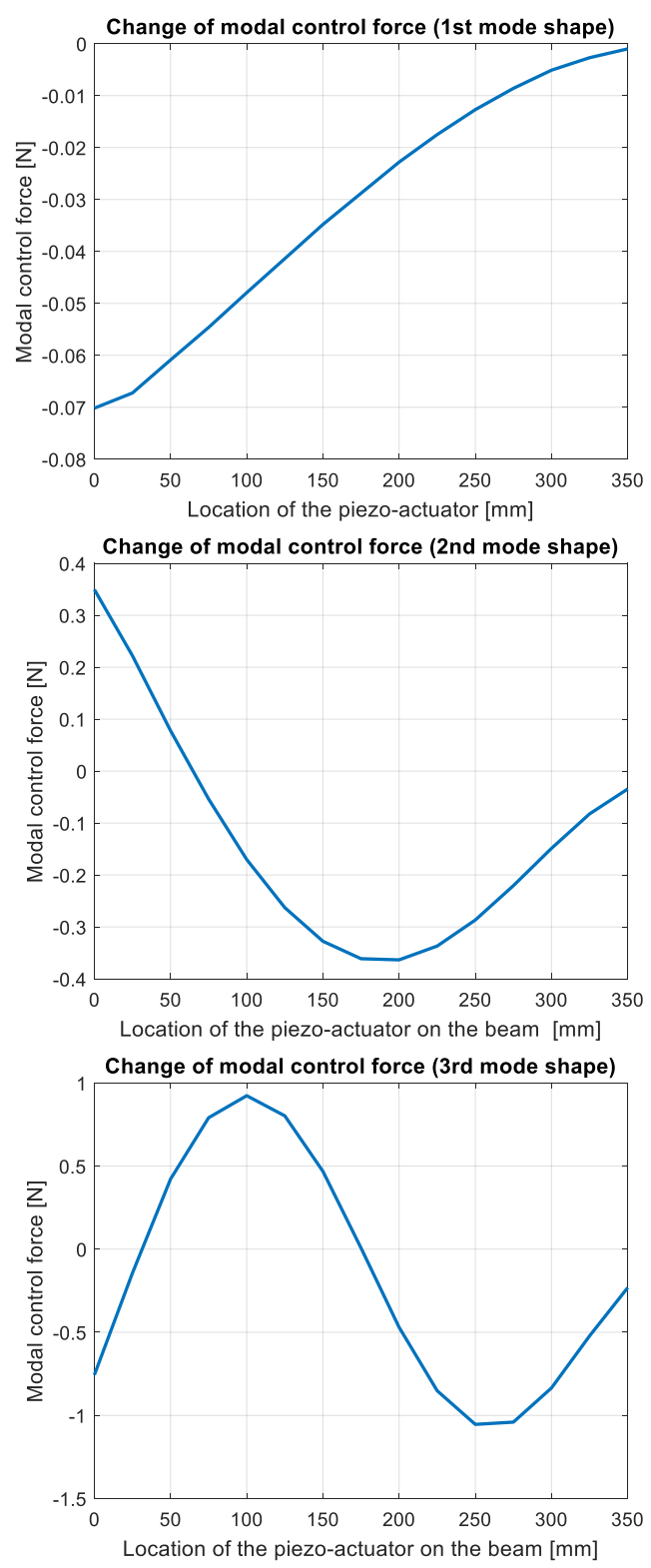

Fig. 4. Influence of the location of the piezo-actuator on modal control force for the first three lowest mode shapes

Taking into account the obtained results shown in Fig.4 it can be noticed that the maximal modal control forces are generated by the piezo-actuator works with the third mode shape but minimal control force when it works with the first mode shapes. Then, 
based on the obtained results can assume that consider piezo will excite beam to vibration in maximal way in vicinity of extremum of the modal control forces. So, in order to achieve maximum voltage from the piezo-harvester located on free end of the beam the piezo-actuator should be located on the beam in distance of $225 \mathrm{~mm}$ from the fixed end. Further experimental investigations carried out at the stand lab are shown in Fig. 5 and allow to verify this actuator location on the beam.

\section{EXPERIMENTAL INVESTIGATIONS}

The active cantilever beam has been experimentally investigated. For this purpose the laboratory stand consists of a steel beam with piezo-actuator QP20N, piezo harvester V21BL with energy harvester power condition system EHE004 is used. In order to drive the piezo-actuator the bipolar amplifier Piezomechanik SVRbip/150 is used. On the other hand in order to measure the vibration of the beam the laser sensor LQG1065PUQ is used.

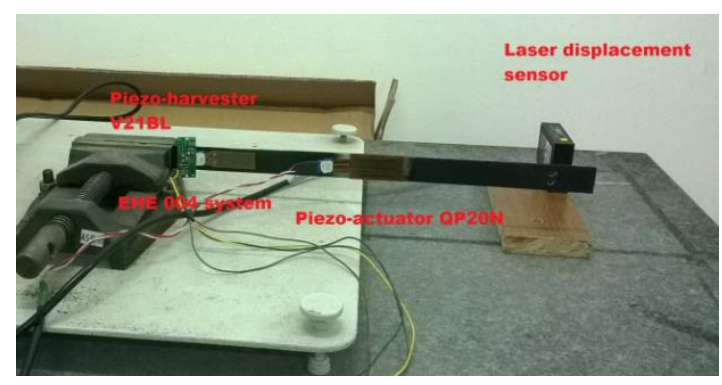

Fig. 5. Photo of the stand lab with the piezoelectric beam

The experimental investigations also were carried out in two steps. In the first step the beam was investigated in the frequency domain in order to validate the obtained results in a computer simulation. For this purpose a chirp signal as a signal excitation in following form $u(t)=5 \sin (\omega t)$ in selected frequency range from $5 \mathrm{~Hz}$ to $450 \mathrm{~Hz}$ is generated from Digital Signal Analyzer (DSA). Then, the amplified periodic signal $u(t)$ is applied to the piezo-stripe actuator and in the same time the vibration of the beam is measured by the laser sensor. As a result of these investigations the frequency response of the beam is obtained which is shown in Fig. 6 . The connection scheme of the laboratory stand during frequency response measurement is shown in Fig. 7.

Taking into account the obtained experimental Bode plot of the piezoelectric beam and Tab. 2 it can be noticed that only first two lowest natural frequencies are close to frequencies with simulations. Therefore, in order to proper excite of the beam to vibration is used analyser DSA which allows to generate sinusoidal signal $\mathrm{u}(\mathrm{t})$ with natural frequency equal $f_{1}=7.07[\mathrm{~Hz}], f_{2}=46.4$ [Hz] or $f_{3}=141.9[\mathrm{~Hz}]$. In results of these excitations the part of mechanical energy is converted to electric energy by used piezoharvester V21B located on free-end of the beam and next converted with $A C$ to $D C$ by an energy harvester power condition (EHPC) system type of EHE004 which is also located on the beam. The scheme of this converter is shown in Fig. 8.

In results of this conversion voltage with $A C$ to $D C$ was possible its measured and recorded. As we can see in Figs. 9-11 the measurements of voltage in the capacitor are carried out for the three lowest natural frequencies of the beam.

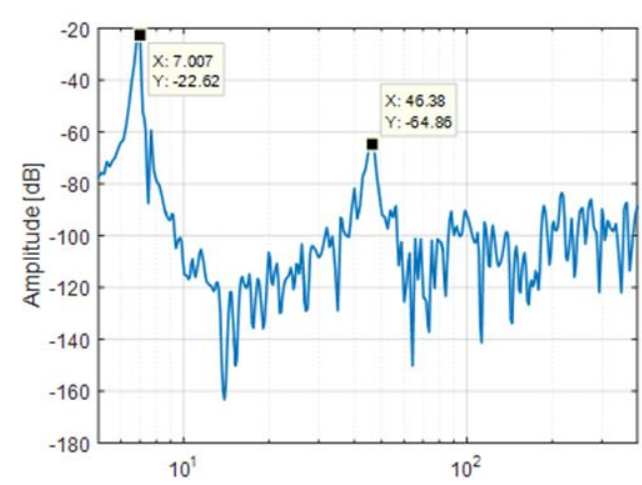

Fig. 6. Experimental Bode plot of the displacement's cantilever beam with the piezo-actuator QP10N and the piezo-harvester V21B

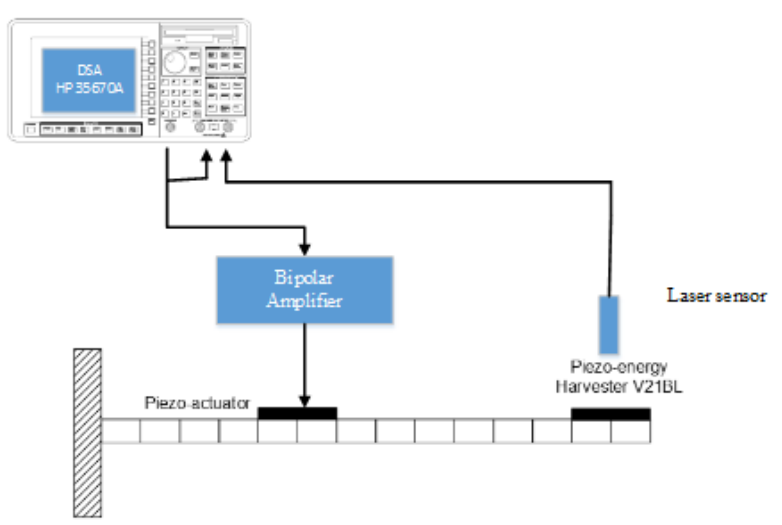

Fig. 7. Schematic diagram of the stand lab to determine modal parameters of the beam

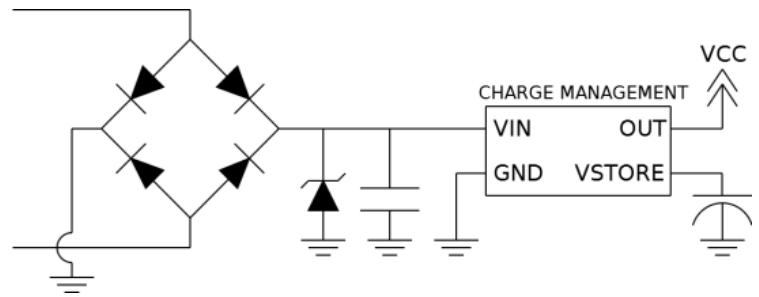

Fig. 8. The energy harvester power condition system type of EHE004. The VIN denotes input voltage, the GND denotes ground, the VSTORE denotes voltage stored in an embedded capacitor $\mathrm{C}=200 \mu \mathrm{F}$, and the OUT - output voltage (VCC)

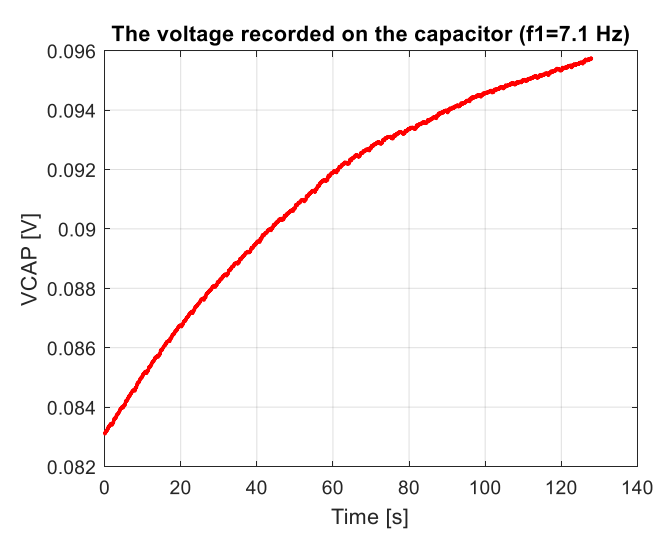

Fig. 9. The voltage stored on the capacitor for the first natural frequency of the beam $\left(f_{1}=7.07 \mathrm{~Hz}\right)$ 


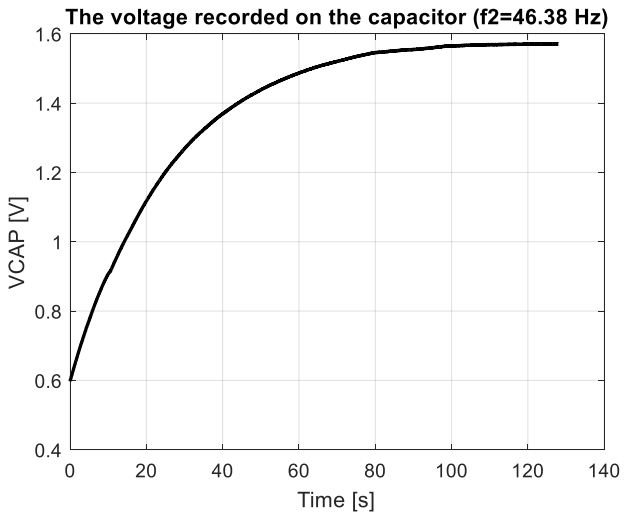

Fig. 10. The voltage stored on the capacitor for the second natural frequency of the beam $\left(f_{2}=46.4 \mathrm{~Hz}\right)$

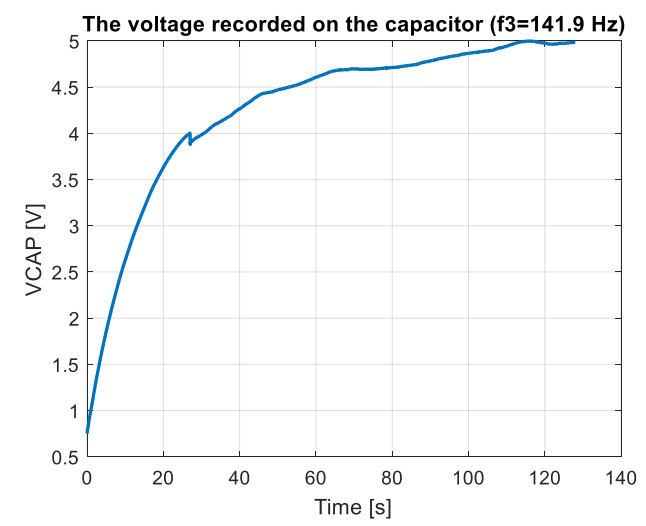

Fig. 11. The voltage stored on the capacitor for third natural frequency of the beam $\left(f_{3}=141.9 \mathrm{~Hz}\right)$

Taking into account recorded voltage it can be noticed that excitation to vibration of the beam with the first natural frequency is unsatisfying, because the measured voltage from piezo harvester very slowly increase to a value of approximately $7 \mathrm{mV}$. Similar behaviour can be seen when the beam is excited to vibration with second natural frequency. Then, the measured voltage on the capacitor achieved only $0.7 \mathrm{~V}$ after long time of oscillation. Finally, the best results are obtained during vibration of the beam with third natural frequency. In this case the recorded voltage from EH element achieved value equals $1.2 \mathrm{~V}$. This level of voltage may supply for example a red LED type diode.

\section{SUMMARY AND CONCLUSION}

The paper shows modelling and testing of the cantilever beam with glued onto its surface two piezoelectric elements work as actuator and harvester. The process of harvesting energy from vibration of the beam was carried out in three steps. In the first step consider piezoelectric beam is analytically analyzed in order to show its electro-mechanical behavior. Next, based on the simulation results the modal parameters of the beam are calculated by using of Ansys software. The obtained results allowed to determining optimal location of the piezo-actuator where the piezo generates maximal modal control forces.

The last step of investigations has been related with experimental testing of the beam on the stand lab. In result of proper excitation of the beam to vibration with using Dynamical Signal Analyzer was possible measure and record the voltage derived from the harvester and verified results obtained in simulations. The results with Figs. 9-11 shown cases achieve of maximal and minimal voltage derived from the capacitor mounted on the EHPC system. Summary obtained results we can notice that exist strongly coupling between mechanical part of the system expressed in form of modal control forces and electrical part - voltage generated by the piezo-harvester. Approach described in the paper leads to design of vibration control system with using fuzzylogic controller which ensures obtain the highest indicator of harvesting energy from excited to vibration the beam in selected frequency range. Results of this investigations will describe in further paper.

\section{REFERENCES}

1. Anton S.R., Sodano H.A. (2007), A review of power harvesting using piezoelectric materials, Smart Materials and Structures, 16, $1-21$.

2. Arnold D. (2007), Review of microscale magnetic power generation, IEEE Transactions on Magnetics, 43, 3940-3951.

3. Bai Y., Havranek P., Tofel P., Button T.W. (2015) Nonlinear piezoelectric devices for broadband air-flow energy harvesting, The European Physical Journal Special Topics, 224, 2675-2685.

4. Borowiec M. (2015), Energy harvesting of cantilever beam system with linear and nonlinear piezoelectric model, The European Physical Journal Special Topics, 224, 2771-2785.

5. Chen S.N., Wang G.J., Chien M.C. (2006), Analytical modeling of piezoelectric vibration-induced micro power generator, Mechatronics, 16, 387-397.

6. Friswell M.I., Ali S.F., Adhikari S., Lees A.W., Bilgen O., Litak G. (2012), Nonlinear piezoelectric vibration energy harvesting from a vertical cantilever beam with tip mass, Journal of Intelligent Material Systems and Structures, 23, 1505-1521.

7. Gosiewski Z., Koszewnik A. (2007), Modeling of beam as control plane for vibration control system, Solid State Phenomena, 144, 5964

8. Kelly S.G. (2007), Advanced Vibration Analysis, CRC Press.

9. Koszewnik A., Grześ P., Walendziuk W. (2015), Mechanical and electrical impedance matching in a piezoelectric beam for energy harvesting, The European Physical Journal Special Topics, 224, 2719-2731.

10. Priya S., Inman D.J. (2009), Energy harvesting technologies, Springer Verlag, New York.

11. Roundy S., Wright P.K., Rabaey J. (2002), Micro-electrostatic vibration-to-electricity converters, Proceedings of the ASME 2002 International Mechanical Engineering Congress and Exposition.

12. Sodano H., Inman D., Park G. (2005), Generation and storage of electricity from power harvesting devices, Journal of Intelligent Material Systems and Structures, 16, 67-75.

13. Shu Y.C., Lien I.C. (2006), Analysis of power output for piezoelectric energy harvesting systems, Smart Materials and Structures, 15, 1499-1512.

14. Tan D., Leng Y.G., Gao Y.J. (2015) Magnetic force of piezoelectric cantilever energy harvesters with external magnetic field, The European Physical Journal Special Topics, 224, 2839-2853.

15. Williams C.B., Yates R.B. (1996), Analysis of a micro-electric generator for microsystems, Sensors and Actuators, 52, 8-11.

The work is supported by Ministry Science and Higher Education under the research project No. S/WM/1/2016 